\title{
Generic Conditions for Stable Hybrid Stars
}

\author{
Mark G. Alford ${ }^{1, a}$ and Sophia $\operatorname{HAN}^{1, b}$ \\ ${ }^{1}$ Physics Department, Washington University, St. Louis, MO 63130, USA
}

\begin{abstract}
We study the mass-radius curve of hybrid stars, assuming a single first-order phase transition between nuclear and quark matter, with a sharp interface between the quark matter core and nuclear matter mantle. We use a generic parameterization of the quark matter equation of state, which has a constant, i.e. density-independent, speed of sound ("CSS"). We argue that this parameterization provides a framework for comparison and empirical testing of models of quark matter. We obtain the phase diagram of possible forms of the hybrid star mass-radius relation, where the control parameters are the transition pressure, energy density discontinuity, and the quark matter speed of sound. We find that this diagram is sensitive to the quark matter parameters but fairly insensitive to details of the nuclear matter equation of state.

We calculate the maximum hybrid star mass as a function of the parameters of the quark matter EoS, and find that there are reasonable values of those parameters that give rise to hybrid stars with mass above $2 M_{\odot}$.
\end{abstract}

\section{Introduction}

We parametrize the dense matter equation of state in terms of three parameters: the pressure $p_{\text {trans }}$ of the transition from nuclear matter to quark matter, the discontinuity in energy density $\Delta \varepsilon$ at the transition, and the speed of sound $c_{\mathrm{OM}}$ in the CSS parameterization of quark matter[1]. We assume that there is a first-order phase transition between nuclear and quark matter, and that the surface tension of the interface is high enough to ensure that the transition occurs at a sharp interface (Maxwell construction) not via a mixed phase (Gibbs construction). This is a possible scenario, given the uncertainties in the value of the surface tension. (For analysis of generic equations of state that continuously interpolate between the phases to model mixing or percolation, see Refs. [2, 3].) This "CSS" parameterization can be viewed as the lowest-order terms of a Taylor expansion of the quark matter EoS about the transition pressure,

$$
\varepsilon(p)= \begin{cases}\varepsilon_{\mathrm{NM}}(p) & p<p_{\text {trans }} \\ \varepsilon_{\mathrm{NM}}\left(p_{\text {trans }}\right)+\Delta \varepsilon+c_{\mathrm{QM}}^{-2}\left(p-p_{\text {trans }}\right) & p>p_{\text {trans }}\end{cases}
$$

where $\varepsilon_{\mathrm{NM}}(p)$ is the nuclear matter equation of state.

The assumption that quark matter has a density-independent speed of sound is reasonably consistent with some well-known quark matter equations of state. For some Nambu-Jona-Lasinio models,

\footnotetext{
ae-mail: alford@wuphys.wustl.edu

be-mail: jhan@physics.wustl.edu
} 
Table 1. Properties of the NL3 and HLPS equations of state. $L$ characterizes the density-dependence of the symmetry energy (see text). NL3 is an example of a stiff EoS, HLPS is an example of a softer one at density $n \lesssim 4 n_{0}$.

\begin{tabular}{lccc}
\hline EoS & max mass & radius at $M=1.4 M_{\odot}$ & $L$ \\
\hline NL3 & $2.77 M_{\odot}$ & $14.92 \mathrm{~km}$ & $118 \mathrm{MeV}$ \\
HLPS & $2.15 M_{\odot}$ & $10.88 \mathrm{~km}$ & $33 \mathrm{MeV}$ \\
\hline
\end{tabular}

the CSS EoS fits Eq. (1) almost exactly [4-7]. In addition, the perturbative quark matter EoS [8] has roughly density-independent $c_{\mathrm{QM}}^{2}$, with a value around 0.2 to 0.3 , above the transition from nuclear matter (see Fig. 9 of Ref. [9]). In the quartic polynomial parameterization [10], varying the coefficient $a_{2}$ between $\pm(150 \mathrm{MeV})^{2}$, and the coefficient $a_{4}$ between 0.6 and 1 , and keeping $n_{\text {trans }} / n_{0}$ above $1.5\left(n_{0} \equiv 0.16 \mathrm{fm}^{-3}\right.$ is the nuclear saturation density), one finds that $c_{\mathrm{OM}}^{2}$ is always between 0.3 and 0.36 . In this paper we study hybrid stars for a range of values of $c_{\mathrm{QM}}^{2}$, from $1 / 3$ (characteristic of very weakly interacting massless quarks) to 1 (the maximum value consistent with causality). We expect that this will give us a reasonable idea of the likely range of outcomes for realistic quark matter.

We use two examples of nuclear EOS: a relativistic mean field model, labelled NL3 [11], and a non-relativistic potential model with phenomenological extrapolation to high density, corresponding to "EoS1" in Ref. [12], labelled HLPS. Some of the properties of HLPS and NL3 are summarized in Table 1 , where $L$ is related to the derivative of the symmetry energy $S_{2}$ with respect to density at the nuclear saturation density, $L=\left.3 n_{0}\left(\partial S_{2} / \partial n\right)\right|_{n_{0}}$. HLPS is a softer equation of state, with a lower value of $L$ and lower pressure at a given energy density (up to $p \approx 3 \times 10^{9} \mathrm{MeV}^{4}, n \approx 5.5 n_{0}$ where its speed of sound rises above 1 and becomes unphysical). NL3 is a stiffer EoS, with higher pressure at a given energy density (also, its speed of sound is less than 1 at all pressures). It yields neutron stars that are larger, and can reach a higher maximum mass.

\section{Criterion for stable hybrid stars}

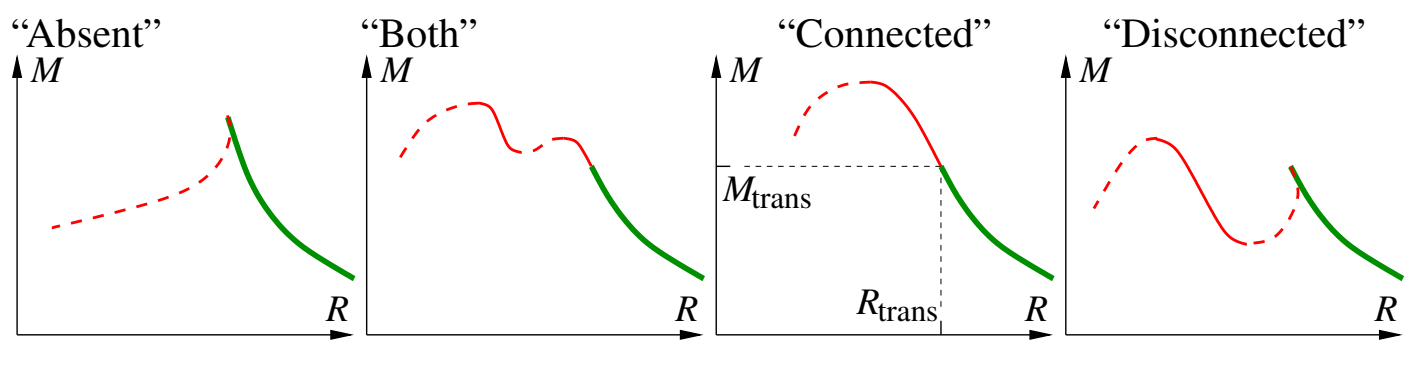

(a)

(b)

(c)

(d)

Figure 1. Schematic form of possible mass-radius relations for hybrid stars. The thick (green) line is the hadronic branch. Thin solid (red) lines are stable hybrid stars; thin dashed (red) lines are unstable hybrid stars. In (a) the hybrid branch is absent. In (c) there is a connected branch. In (d) there is a disconnected branch. In (b) there are both types of branch. In realistic neutron star $M(R)$ curves the cusp that occurs in cases (a) and (d) is much smaller and harder to see $[14,15]$ 
A compact star will be stable as long as the mass $M$ of the star is an increasing function of the central pressure $p_{\text {cent }}[13]$. By performing an expansion in powers of the size of the quark matter core, one can show [14-16] that there is a threshold value $\Delta \varepsilon_{\text {crit }}$ given by

$$
\frac{\Delta \varepsilon_{\text {crit }}}{\varepsilon_{\text {trans }}}=\frac{1}{2}+\frac{3}{2} \frac{p_{\text {trans }}}{\varepsilon_{\text {trans }}}
$$

(This is $\lambda_{\text {crit }}-1$ in the notation of Ref. [14].) In Fig. 1, panels (b) and (c) show possible forms of $M(R)$ for $\Delta \varepsilon<\Delta \varepsilon_{\text {crit }}$, and panels (a) and (d) show possible forms for $\Delta \varepsilon>\Delta \varepsilon_{\text {crit. }}$. In $M(R)$ curves for realistic neutron star equations of state, the cusp at high $\Delta \varepsilon$ is much less clearly visible: the region where the slopes of hybrid and neutron stars match is very small, covering a range in $M$ of less than one part in a thousand near the transition point $\left(R_{\text {trans }}, M_{\text {trans }}\right)$.

In Figs. 1(b) and (d), we illustrate a second, disconnected, branch of stable hybrid stars at $\Delta \varepsilon>$ $\Delta \varepsilon_{\text {crit }}$. This possibility was noted in Ref. [14]. The disconnected branch is a "third family" [17, 18] of compact stars besides neutron stars and white dwarfs. In this paper we will study the generic features of a quark matter EoS that give rise to this phenomenon.

\section{3 "Phase diagram" for hybrid stars}

\subsection{Phase diagram at fixed $c_{\mathrm{QM}}$}
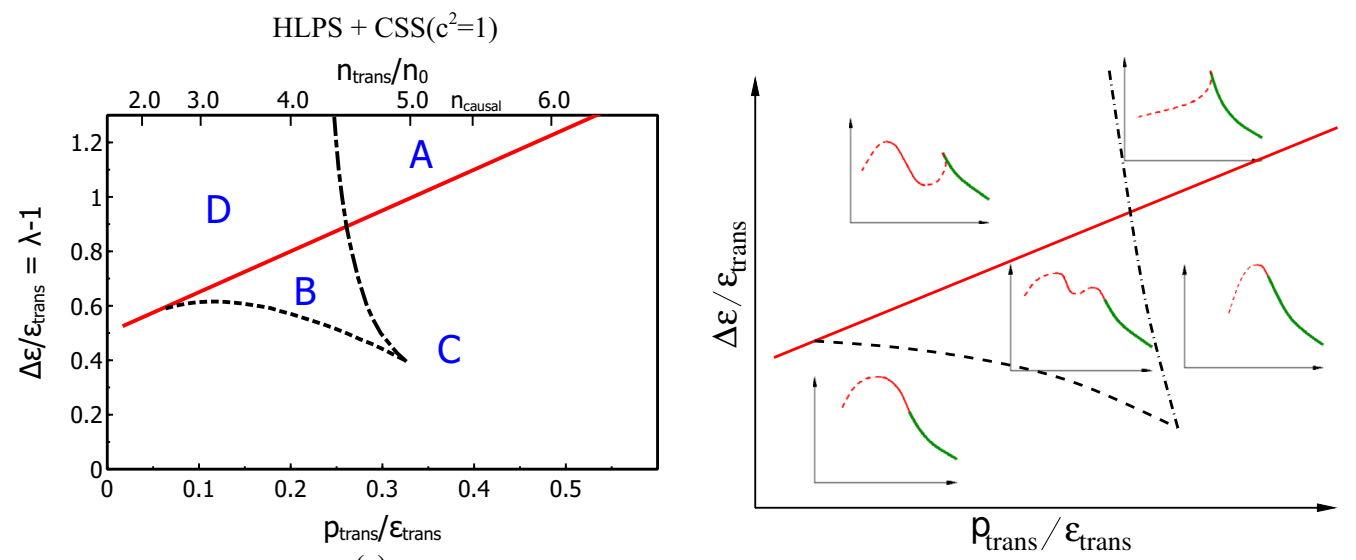

(a)

Figure 2. Phase diagram for hybrid star branches in the mass-radius relation of compact stars.

In Fig. 2 we plot a "phase diagram" for hybrid stars, The left panel of Fig. 2 is the result of calculations for the HLPS nuclear matter EoS and quark matter with $c_{\mathrm{QM}}^{2}=1$. The right panel is a schematic showing the form of the mass-radius relation in each region of the diagram. The regions correspond to different geometries of the hybrid branch displayed in Fig. 1: A="Absent", $\mathrm{C}=$ ="Connected", $\mathrm{D}=$ ="Disconnected", $\mathrm{B}=$ "Both" (connected and disconnected).

The solid straight (red) line is $\Delta \varepsilon_{\text {crit }}$ from Eq. (2). Below the line in regions $\mathrm{B}$ and $\mathrm{C}$, there is a hybrid star branch connected to the nuclear star branch. Above the line in regions A and D, there is no connected hybrid star branch. In regions B and D there is a disconnected hybrid star branch. 
The roughly vertical dash-dotted curve in Fig. 2 marks a transition where an additional disconnected branch of hybrid stars appears/disappears. When one crosses this line from the right, going from region A to D by decreasing the nuclear/quark transition density, a stationary point of inflection appears in $M\left(p_{\text {cent }}\right)$ at $p_{\text {cent }}>p_{\text {trans }}$. If one crosses from $\mathrm{C}$ to $\mathrm{B}$ then this point of inflection is at lower central pressure than the existing maximum in $M\left(p_{\text {cent }}\right)$. This produces a stationary point of inflection in the $M(R)$ relation to the left of the existing maximum (if any). After crossing the dash-dotted line the point of inflection becomes a new maximum-minimum pair (the maximum being further from the transition point), producing a disconnected branch of stable hybrid stars in regions B and D. Crossing the other way, by increasing the transition pressure, the maximum and minimum that delimit the disconnected branch merge and the branch disappears.

The roughly horizontal dashed curve in Fig. 2 which separates region B and C marks a transition between mass-radius relations with one connected hybrid star branch, and those with two hybrid star branches, one connected and one disconnected. Crossing this line from below, by increasing the energy density discontinuity, a stationary point of inflection in $M\left(p_{\text {cent }}\right)$ (or equivalently in $M(R)$ ) appears in the existing connected hybrid branch. Crossing in to region $\mathrm{B}$, this point of inflection becomes a new maximum-minimum pair, so the connected hybrid branch is broken in to a smaller connected branch and a new disconnected branch. The maximum of the old connected branch smoothly becomes the maximum of the new disconnected branch. If one crossed the dashed line in the opposite direction, from $\mathrm{B}$ to $\mathrm{C}$, the maximum closest to the transition point would approach the minimum and they would annihilate, leaving only the more distant maximum.

Where the horizontal and vertical curves meet, the two maxima and the minimum that are present in region $\mathrm{B}$ all merge to form a single flat maximum where the first three derivatives of $M(R)$ are all zero.

\subsection{Varying $c_{\mathrm{QM}}$ and the nuclear EoS}

In Fig. 3 we show the effects of varying the speed of sound and the nuclear matter EoS. Fig. 3(a) is the phase diagram with $c_{\mathrm{QM}}^{2}=1 / 3$, and Fig. 3(b) is for $c_{\mathrm{QM}}^{2}=1$. In both panels we show the phase diagram for HLPS and NL3 nuclear matter EoSs. The straight line is independent of $c_{\mathrm{QM}}^{2}$ and the detailed form of the nuclear matter EoS. The other phase boundaries, outlining the region where there is a disconnected hybrid branch, are remarkably insensitive to the details of the nuclear matter EoS but depend strongly on the quark matter speed of sound. For a given nuclear matter EoS the hybrid branch structure is determined by $p_{\text {trans }} / \varepsilon_{\text {trans }}, \Delta \varepsilon / \varepsilon_{\text {trans }}$, and $c_{\mathrm{QM}}^{2}$, so one could make a three-dimensional plot with $c_{\mathrm{QM}}^{2}$ as the third axis, but this figure adequately illustrates the dependence on $c_{\mathrm{QM}}^{2}$. We will now discuss the physics behind the shape of the phase boundaries.

\subsection{Physical understanding of the phase diagram}

The main feature of the phase diagram is that a disconnected branch is present when the transition density is sufficiently low, and the energy density discontinuity is sufficiently high. It occurs more readily if the speed of sound in quark matter is high. Such features were noticed in the context of stars with mixed phases in Ref. [2], which pointed out that they can be understood as follows.

When a very small quark matter core is present, its greater density creates an additional gravitational pull on the nuclear mantle. If the pressure of the core can counteract the extra pull, the star is stable and there is a connected hybrid branch. If the energy density jump is too great, the extra gravitational pull is too strong, and the star becomes unstable when quark matter first appears. However, if the energy density of the core rises slowly enough with increasing pressure (i.e. if $c^{2}=d p / d \varepsilon$ is large 


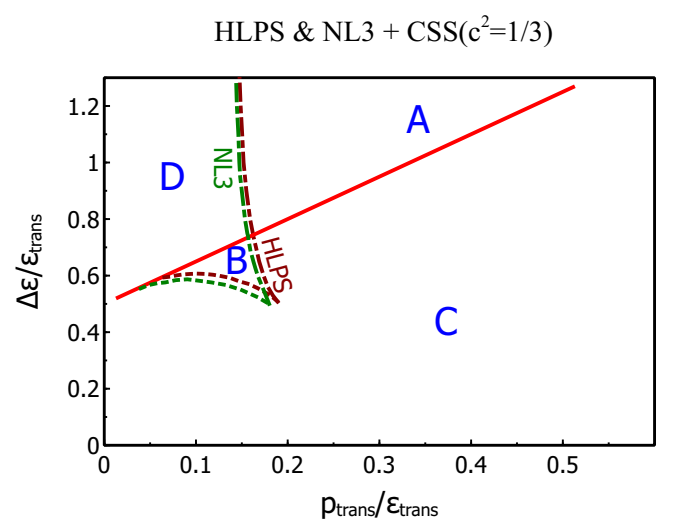

(a)

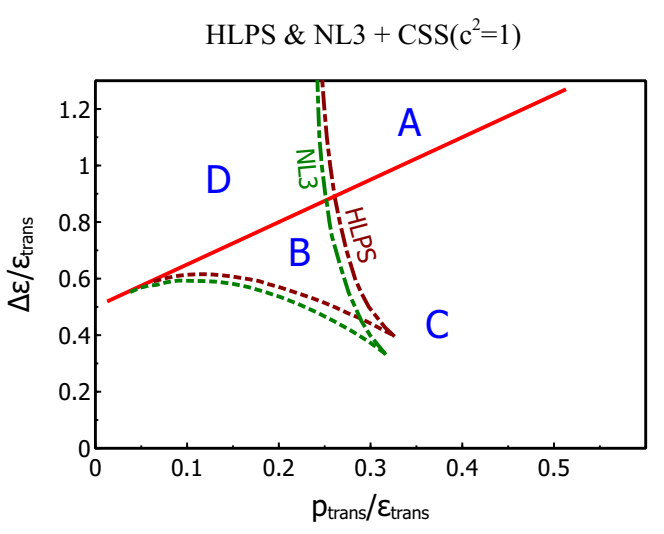

(b)

Figure 3. Phase diagram like Fig. 2, showing that the phase boundaries are not very sensitive to changes in the nuclear matter EoS, but they are affected by varying the quark matter speed of sound.

enough), a larger core with a higher central pressure may be able to sustain the weight of the nuclear mantle above it. Region B, with connected and disconnected branches, is more complicated and we do not have an intuitive explanation for it.

We can now understand why the vertical line marking the $\mathrm{B} / \mathrm{C}$ and $\mathrm{D} / \mathrm{A}$ boundaries moves to the right as $c_{\mathrm{QM}}^{2}$ increases. Since $c^{2}=d p / d \varepsilon$, if $c_{\mathrm{QM}}^{2}$ is larger then the energy density of the core rises more slowly with increasing pressure, which minimizes the tendency for a large core to destabilize the star via its gravitational attraction. Finally, we can see why that line has a slight negative slope: larger $\Delta \varepsilon$ makes the quark core heavier, increasing its pull on the nuclear mantle, and making the hybrid star more unstable against collapse.

\subsection{Observability of hybrid branches}

In Fig. 4 we show the phase diagram for HLPS and NL3 nuclear matter, with $c_{\mathrm{QM}}^{2}=1$ quark matter, with contours showing the measure $\Delta M$ of the length of the hybrid branch, which is the difference in mass between the heaviest hybrid star and the hadronic star just before quark matter appears (whose mass is $\left.M_{\text {trans }}\right)$. At the horizontal boundary between region $\mathrm{C}$ and $\mathrm{B}$, the maximum of the connected branch smoothly becomes the maximum of the disconnected branch (see Fig. 2) so the dashed contours (for the connected branch) connect smoothly to the dot-dashed contours (for the disconnected branch). We see that the $\Delta M$ is roughly independent of details of the nuclear matter EoS, except at high transition pressure where the transition to quark matter is happening close to the maximum of the nuclear EoS, which greatly suppresses the length of the connected branch. Note that although we only show contours for positive $\Delta M$, contours that end on the $\mathrm{A} / \mathrm{D}$ or near-vertical $\mathrm{B} / \mathrm{C}$ boundary can have negative $\Delta M$.

From Fig. 4 we conclude that Eq. (2) is not a good guide to the presence of observable hybrid branches. The connected branch may be very small and hard to detect, even at values of $\Delta \varepsilon / \varepsilon_{\text {trans }}$ well below the critical value of Eq. (2). For the $c_{\mathrm{QM}}^{2}=1$ case, only in the parts of region $\mathrm{C}$ that lie approximately below region $\mathrm{B}$ (i.e. for $p_{\text {trans }} / \varepsilon_{\text {trans }} \lesssim 0.3$ ) is the connected hybrid branch large enough 


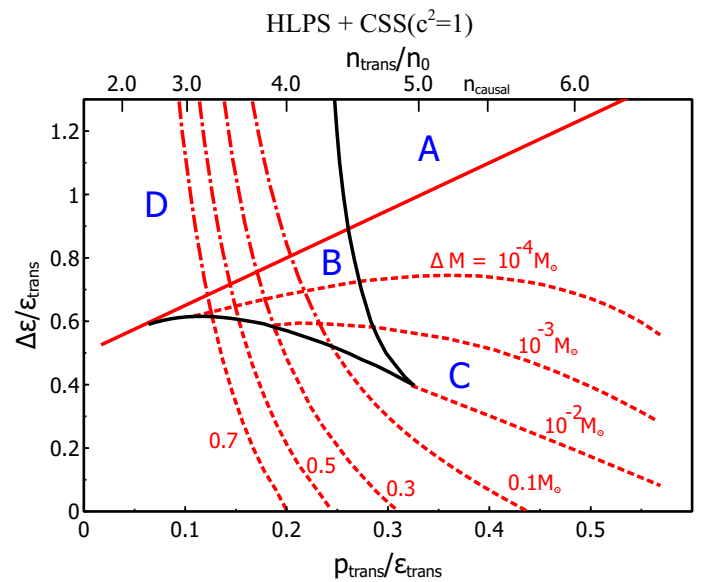

(a)

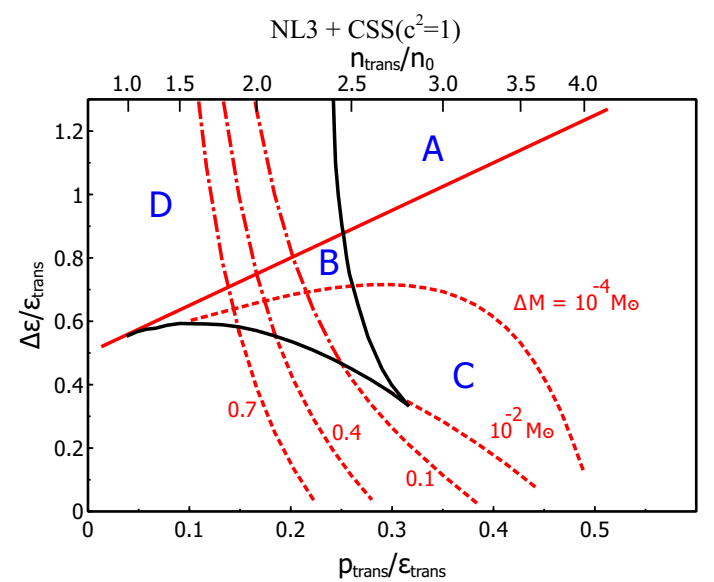

(b)

Figure 4. Contour plot of a measure of the observability of hybrid branches: $\Delta M$.

to be detectable via observations of mass that have an experimental uncertainty of around $0.01 M_{\odot}$. When a disconnected branch is present, the connected branch is either absent (region D) or too small to observe (region B).

\section{Maximum mass of hybrid stars}

Using the CSS parameterization (1) of the quark matter EoS, it is possible to get hybrid stars that are heavy enough to be consistent with recent measurements of stars of mass $2 M_{\odot}[19,20]$. In Fig. 5 we show contour plots for the maximum masses of hybrid stars on the ( $\left.p_{\text {trans }} / \varepsilon_{\text {trans }}, \Delta \varepsilon / \varepsilon_{\text {trans }}\right)$ parameter space of the quark matter EoS at fixed $c_{\mathrm{QM}}^{2}$, for a soft nuclear equations of state Bruecker-Hatree-Fock (BHF) [21]. The shaded region inside the $M_{\max }=2 M_{\odot}$ contour on the parameter space is eliminated by the observational constraint.

The dot-dashed (red) contours are for hybrid stars on a connected branch, while the dashed (blue) contours are for disconnected branches. When crossing the near-horizontal boundary from region $\mathrm{C}$ to $\mathrm{B}$ or $\mathrm{D}$ the connected branch becomes a smaller connected branch and a disconnected branch, so the maximum of the connected branch smoothly becomes the maximum of the disconnected branch. The red contour in the $\mathrm{C}$ region smoothly becomes a blue contour in the $\mathrm{B}$ and $\mathrm{D}$ regions, and finally ends on the near-vertical boundary. When crossing the near-vertical boundary form region $\mathrm{C}$ to $\mathrm{B}$, the small connected branch does not change so the red dashed contour continues until it hits the near-horizontal boundary.

On the right side of each panel, the connected branch (red dashed) contours are almost vertical, corresponds to a very small connected hybrid branch which exists in a very small range of central pressures above $p_{\text {trans }}$, beyond which they become unstable. The maximum mass on this branch is therefore very close to the mass of the heaviest purely-nuclear matter star (the one for $p_{\text {cent }}=p_{\text {trans }}$ ) which is independent of the quark matter parameters $\Delta \varepsilon$ and $c_{\mathrm{QM}}^{2}$, hence the contour is vertical. These hybrid stars have a tiny quark matter core and cover a tiny range of masses, and so would be very hard to observe. 

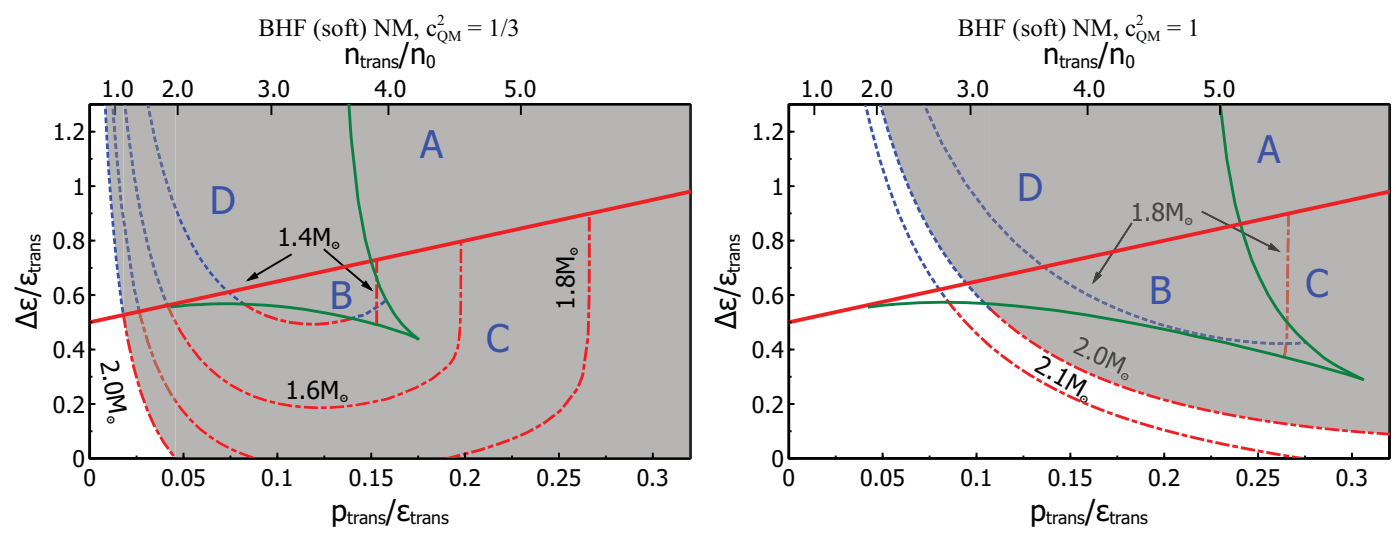

Figure 5. Contour plot of the mass of the heaviest hybrid star as a function of quark matter EoS parameters.

We see that for quark matter with $c_{\mathrm{QM}}^{2}=1$ (right panel), the eliminated region is not very large, which means a reasonable range of the other two parameters, $p_{\text {trans }} / \varepsilon_{\text {trans }}$ and $\Delta \varepsilon / \varepsilon_{\text {trans }}$, is compatible with the observation. But for quark matter with $c_{\mathrm{OM}}^{2}=1 / 3$ (left panel) as predicted by many models, a large region is excluded and the space available to transition density and energy density discontinuity is fairly small $[22,23]$. For a stiffer nuclear equations of state Dirac-Bruecker-Hatree-Fock (DBHF) [24], we found that the parameter space is less constrained.

\section{References}

[1] For a classical ideal gas, the squared speed of sound $c_{s}^{2} \propto T / m$, where $T$ is the temperature and $m$ is the mass, and is independent of density. For a quantum ideal gas, $c_{s}^{2}=(1 / 3)\left(1-(\mathrm{m} / \mu)^{2}\right)$, where $\mu$ is the chemical potential inclusive of mass, and varies between $1 / 3$ (ultra-relativistic case) and $\sim(2 / 3) n^{2 / 3}$ (the non-relativistic case), where $n$ is the number density.

[2] Macher, Jean and Schaffner-Bielich, Jurgen: Eur.J.Phys. 26, 341 (2005)

[3] Masuda, Kota and Hatsuda, Tetsuo and Takatsuka, Tatsuyuki: ApJ. 764,12 (2013)

[4] Agrawal, B.K.: Phys.Rev. D81, 023009 (2010)

[5] Bonanno, Luca and Sedrakian, Armen: Astron.Astrophys. 539, A16 (2011)

[6] Lastowiecki, R. and Blaschke, D. and Grigorian, H. and Typel, S.: Acta Phys.Polon.Supp. 5, 535-540 (2011)

[7] Zdunik, J.L. and Haensel, P.: Astron. Astrophys. 551, A61 (2013)

[8] Kurkela, Aleksi and Romatschke, Paul and Vuorinen, Aleksi and Wu, Bin: arxiv:1006.4062 (2010)

[9] Kurkela, Aleksi and Romatschke, Paul and Vuorinen, Aleksi: Phys.Rev. D81, 105021 (2009)

[10] Alford, Mark and Braby, Matt and Paris, M.W. and Reddy, Sanjay: Astrophys.J. 629, 969-978 (2005)

[11] Shen, G. and Horowitz, C.J. and Teige, S.: Phys.Rev. C83,035802 (2011)

[12] Hebeler, K. and Lattimer, J.M. and Pethick, C.J. and Schwenk, A.: Phys.Rev.Lett. 105, 161102 (2010).

[13] Bardeen, J. M. and Thorne, K. S. and Meltzer, D. W.: ApJ. 145, 505 (1966)

[14] Schaeffer, R. and Zdunik, L. and Haensel, P.: Astron. 126, 1804-1828 (1983) 
[15] Lindblom, Lee.: Phys.Rev. D58, 024008 (1998)

[16] Seidov, Z. F.: Sov. Astron. 15, 347 (1971)

[17] Glendenning, N. K. and Kettner, C.: Astron. Astrophys. L9, 353 (2000)

[18] Schertler, K. and Greiner, C. and Schaffner-Bielich, J. and Thoma M. H.: Nucl. Phys. A677, 463 (2000)

[19] Antoniadis, John and Freire, Paulo C.C. and Wex, Norbert and Tauris, Thomas M. and Lynch, Ryan S. and others: Science 340, 6131 (2013)

[20] Demorest, Paul and Pennucci, Tim and Ransom, Scott and Roberts, Mallory and Hessels, Jason: Nature 467, 1081-1083 (2010)

[21] Schulze, H.-J. and Cugnon, J. and Lejeune, A. and Baldo, M. and Lombardo, U.: Phys.Rev. C52, 2785-2788 (1995)

[22] Alford, Mark G. and Han, Sophia and Prakash, Madappa: Phys.Rev. D88, no.8 083013 (2013)

[23] Bedaque, Paulo F. and Steiner, Andrew W.: arXiv: 1408.5116, (2014)

[24] T. Gross-Boelting, C. Fuchs, and A. Faessler, Nucl. Phys. A648, 105-137 (1999) 